\title{
Metodología de evaluación del clima organizacional a través de un modelo de regresión logística para una universidad en Bogotá, Colombia*
}

\section{Work environment evaluation methodology through a logistic regression model for a university in Bogota, Colombia.}

\author{
Juan Camilo Vega** \\ Edgar Guillermo Rodríguez Díaz*** \\ Alexandra Montoya R.****
}

Recibido: 11 de noviembre de 2011

Aprobado: 6 de febrero de 2012

\section{Resumen}

El artículo presenta el desarrollo de una metodología de evaluación de clima organizacional entre diferentes grupos de interés dentro de una organización académica, mediante

* El artículo hace parte de la línea de investigación: "Ambiente laboral en las Organizaciones" de la Universidad Nacional Abierta y a Distancia (UNAD). Cómo citar este artículo: Vega, J., Rodríguez, E., \& Montoya, A. (2012). Metodología de evaluación del clima organizacional a través de un modelo de regresión logística para una universidad en Bogotá, Colombia. Revista CIFE, 14 (21), 247-272.

** MSc, Coordinador Nacional de la Especialización en Gerencia Estratégica de Mercadeo. Docente auxiliar de la Escuela de Ciencias Administrativas, Contables, Económicas y de Negocios de la UNAD. Correo electrónico: juan. vega@unad.edu.co

*** MSc, Vicerrector de Desarrollo Regional y Proyección Comunitaria de la UNAD. Correo electrónico: edgar.rodriguez@unad.edu.co

****PhD, Coordinadora Nacional del Concurso Docente, profesora asociada de la Facultad de Ciencias Económicas de la Universidad Nacional de Colombia. Correo electrónico: lamontoyar@unal.edu.co 


\section{ĊIF́E 21}

ISSN: 0124-3551 / Año 14, No 21 / julio-diciembre / pp. 247-272

la formulación de un modelo de regresión logística. aplicase aplicaron 169 cuestionarios entre el personal de una universidad pública en Colombia con el fin de contrastar los resultados por Sede y Unidad Funcional; el análisis de frecuencias acumuladas muestra, a nivel general, calificaciones bajas referentes a la remuneración y el reconocimiento laboral pero al comparar los resultados obtenidos en la aplicación del modelo de regresión logística se infieren posiciones diferentes en estos aspectos entre administrativos y docentes, los maestros perciben un bajo reconocimiento de su trabajo a pesar de sentirse bien remunerados, contrario a lo que sucede con el personal administrativo.

Palabras claves: clima organizacional, modelo de regresión logística, educación superior.

Clasificación JEL: M10, M54

\section{Abstract}

This article develops an assessment methodology of the organizational climate among different of stakeholders inside an academic organization through a model formulation of logistic regression. In this sense, there were applied 169 questionnaires in the staff of a public university in Colombia in order to contrast with the results by branch and functional department. The analysis of accumulated frequencies show low grades regarding remuneration and labor recognition on a general level; although; to contrast the obtained results of logistic regression's model that allows deducing different positions in these aspects between the administrative and teachers' staff. By this means that academic staff perceives a low recognition in their work in spite of having a good remuneration, otherwise happens with the administrative staff.

Keywords: organizational climate, logistic regression's model and higher education.

Classification JEL: M10, M54 
Metodología de evaluación del clima organizacional a través de un modelo de regresión logística para una universidad en Bogotá, Colombia

Juan Camilo Vega, Edgar Guillermo Rodríguez Díaz, Alexandra Montoya R.

\section{Introducción}

En la actualidad existen varios instrumentos para evaluar el clima organizacional que miden aspectos como: liderazgo, motivación, autonomía, toma de decisiones, estructura organizacional, remuneración, recompensas, relaciones interpersonales, entre otros, sin embargo los resultados son analizados mediante tablas de frecuencia de calificación que solo permiten una valoración a nivel global y por variable, en la que los análisis se centran en una comparación con un estándar de rangos de puntuación previamente establecido por el investigador. El estudio emplea un modelo de regresión logística que permite identificar la cantidad y las características de correlación entre diferentes unidades funcionales, grupos o sedes respecto a las variables evaluadas en el instrumento, convirtiéndose en una herramienta válida para la toma decisiones efectivas en áreas específicas de la organización y encontrar las ventajas o debilidades de estas frente a la percepción del ambiente laboral.

En Colombia los trabajos más reconocidos sobre clima organizacional son los realizados por Méndez (2006) y Toro (2009), que abordan estudios de multirrelación entre empresas de un mismo sector. La investigación que se presenta se desarrolla dentro del marco de uno de estos y toma como objeto de análisis una universidad pública con diferentes sedes en el país y con una estructura funcional que distingue dos grandes categorías: las funcionales y las misionales; con estos parámetros, soportados en los análisis de frecuencia y complementados por el análisis a través de la formulación de un modelo de regresión logística, se compara la percepción laboral entre las sedes y las áreas funcionales.

\section{Antecedentes sobre estudios de clima organizacional}

\subsection{Definición}

El clima organizacional se define desde distintas posturas como una variable mediadora entre las realidades sociales y orgánicas de la empresa y la conducta individual (Toro 2009, p. 17, Calderón, et al. 2006); también se describe como un conjunto de atributos específicos de un órgano particular que refleja la manera en que se establecen las relaciones entre la organización, sus miembros y el medio ambiente (Marín, 2003, p. 21, McMurray, Scott, 2003); en otra postura, el clima es una variable que incide en la satisfacción y motivación de los individuos (Salinas, et al, 1994, pp. 22-29), y otros autores, por el contrario, lo determinan un resultado de los procesos de socialización de los sujetos (Schneider, Reichers, 1983, p. 31 Pérez, et al 2006), en esta línea el clima también es influenciado por otros factores como el comportamiento administrativo, las condiciones organizacionales que perciben los individuos y también por sus informaciones, capacidades, esperanzas, valores y percepciones (Marín, 2003, p. 22 ); en otro extremo existen planteamientos que lo presentan a manera de una variable independiente del 


\section{ĊIF́E 21}

ISSN: 0124-3551 / Año 14, No 21 / julio-diciembre / pp. 247-272

individuo o del entorno, inherente a la organización y definida como: "El conjunto de características permanentes que describen una organización, la distinguen de otra, e influyen en el comportamiento de las personas que la forman" (Forehand y Gilmer 1964, p. 362) por lo cual es fruto de la estructura organizacional (Ashkanasy, 2008) y se centra en la distribución funcional, la formalización de las políticas y las reglas que restringen la conducta individual; sin embargo las definiciones más aceptadas de este término son las que buscan integrar las características estructurales de la organización, los procesos de interacción de los individuos y la percepción de los individuos, al respecto Méndez (2006, pp. 35-36) incorpora estos elementos explicando el clima organizacional como la suma de características de la organización formal e informal, las cuales al ser percibidas por el individuo determinan su comportamiento en actitudes que se reflejan en sus niveles de motivación por lo cual un análisis de este debería orientarse en torno a las siguientes particularidades:

- Los aspectos que identifican el sistema social: liderazgo, relaciones interpersonales, cooperación, objetivos, toma de decisiones, motivación y control.

- Los aspectos subjetivos que el individuo construye en el proceso de interacción social con el líder, los compañeros y la organización, que se constituyen con base en la experiencia, las creencias, las percepciones, la participación y la actitud.

- La capacidad del individuo de asimilar sus percepciones de clima organizacional debido al carácter dinámico de la organización.

- La incidencia que tiene la construcción del clima organizacional individual en aspectos como la satisfacción en el trabajo y la productividad, lo cual puede modificar los grados de eficiencia y de productividad.

Un análisis del clima organizacional visto desde esta perspectiva permite identificar las fortalezas y los aspectos a mejorar para lograr un ambiente de trabajo que facilite un mejor desempeño del individuo (Segredo, Pérez, 2007) y alcanzar los objetivos particulares y los de la organización.

Los estudios de clima organizacional según Dancerau, Alutto y Yammarino (citado por Toro, 2006, p. 69) se clasifican en:

- Un solo nivel (entre persona, grupo y colectivo).

- Multinivel: combinación de varios de los anteriores.

- Multivariable: estudio simultáneo de varios factores o variables.

- Multirrelación: evaluación de semejanzas y diferencias entre grupos de una organización o entre instituciones. 
Un buen clima organizacional permite aumentar el rendimiento de la empresa, e influye positivamente en el comportamiento de los trabajadores, puesto que puede significar que estos se sientan identificados e integrados en la estructura de la organización (Pérez, Tejada et al., 2007, pág. 2), al respecto existe un consenso entre los diferentes investigadores que han abordado el tema, al considerar el clima como "la percepción colectiva y compartida de las realidades internas del grupo "(Toro, 2009, p. 68); en este orden de ideas: "El comportamiento de la gente está basado en la percepción de lo que es la realidad, no en la realidad misma" (García 2007, p. 155), es decir, "el mundo en la manera en que es percibido por el individuo es lo que realmente importa para el comportamiento" (García, 2007, p. 155) por lo cual "las percepciones son representaciones cognitivas de la realidad y estas son las precursoras de los juicios y las actuaciones de las personas" (Toro, 2009, p. 70), de donde se infiere que una modificación en la percepción del individuo con respecto a su trabajo ocasionará cambios de comportamiento frente al mismo. La conducta de un sujeto en su trabajo se define en dos aspectos; el primero, relacionado con características propias de la persona implicada y con su entorno (Brunet, 1987, p.11) donde el comportamiento del individuo en determinada situación laboral depende de particularidades dinámicas e interdependientes, relacionadas con el individuo como las actitudes, las aptitudes, los rasgos psicológicos y físicas; y el segundo, fundamentado en la percepción de las estructuras de la organización (Goncalves, 2010, p. 2, Ucros, 2011). De la integración de estos elementos se obtiene el clima organizacional (Brunet 1987, p. 12).

\subsection{Medición}

Para emprender una investigación de clima organizacional cimentada en lo mencionado, es importante definir que una organización está compuesta por una serie de variables (Alcántar, et al. 2012), calificadas por los individuos, que determinan el clima e influyen en el comportamiento de sus miembros. El clima organizacional es un componente multidimensional establecido por la estructura organizacional, el tamaño de la organización, los modos de comunicación, los estilos de dirección, entre otros (Caligiori y Díaz, 2003, p. 645). Y que se pueden asociar en dos grupos: estructura organizacional y procesos organizacionales (Brunet, 1987, p. 15). La estructura organizacional corresponde a las diferentes formas en las cuales se puede dividir el trabajo y coordinarlo para alcanzar el logro de los objetivos; está conformada por tres aspectos (Hutt y Marmiroli, 2007, p. 1):

1. Los mecanismos de control: hacen referencia a todos los instrumentos de control y de poder sobre las tareas que establece la organización, entre ellas la supervisión directa, el autocontrol, y la estandarización de procesos de trabajo y de resultados.

2. La división del trabajo: se enfoca en observar la organización dividida por sectores y en ella identifica cinco componentes básicos, según la característica del trabajo 


\section{ĊIF́E 21}

ISSN: 0124-3551 / Año 14, No 21 / julio-diciembre / pp. 247-272

que realizan los miembros de la organización, estos son de núcleo básico (funciones operativas), cumbre estratégica (los miembros que manejan la organización y los de apoyo a estos), línea media (miembros de la organización que sirven de nexo entre los de núcleo básico y los de cumbre estratégica), tecno-estructura (miembros que se enfocan en la estandarización de procesos) y órganos o personal de apoyo.

3. El funcionamiento de la organización: aquí se reconocen las distintas formas de jerarquización y autoridad, desde la implantada de manera formal hasta las que surgen del trabajo e interacción entre las distintas áreas.

Los procesos organizacionales están relacionados con la gestión de recursos humanos y se pueden encontrar, entre otros, el liderazgo, la comunicación, el control, la gestión de conflictos, la coordinación, la toma de decisiones, la especialización de las funciones, las relaciones entre los individuos, los mecanismos de socialización entre los individuos y el grado de autonomía de los empleados (Brunet, 1987, p. 15).

Una evaluación del clima organizacional debe incluir una conjugación de variables relacionadas con la percepción de los miembros de la organización respecto a la estructura y los procesos organizacionales. Las investigaciones sobre el tema se soportan, principalmente, en la obtención de información primaria, directamente de los individuos que conforman la organización, mediante la aplicación de un cuestionario ( Méndez, 2006, p. 53); los investigadores de clima suelen utilizar los cuestionarios para desarrollar estudios comparativos y longitudinales ( Brunet, 1987, pág. 42), los primeros, como los realizados por Toro (2009) y Méndez (2006), hacen un comparativo del clima organizacional en distintas organizaciones en Colombia, mientras que en los segundos se evalúan los efectos del clima organizacional en una institución específica. En la tabla 1, se presenta un recuento de las distintas investigaciones enfocadas en el diseño e implementación de cuestionarios que sirven como referente para la construcción de instrumentos para evaluar el clima organizacional. 
Metodología de evaluación del clima organizacional a través de un modelo de regresión logística para una universidad en Bogotá, Colombia

Juan Camilo Vega, Edgar Guillermo Rodríguez Díaz, Alexandra Montoya R.

Tabla 1. Instrumentos para evaluar clima organizacional

\begin{tabular}{|c|c|c|}
\hline Autor & Propósito & Dimensiones \\
\hline $\begin{array}{l}\text { Friedlander y Mar- } \\
\text { gulies (1969, pp. } \\
171-183)\end{array}$ & $\begin{array}{l}\text { Medir el impacto del clima } \\
\text { organizacional y de los } \\
\text { valores organizacionales en la } \\
\text { satisfacción } \\
\text { en el trabajo. }\end{array}$ & $\begin{array}{l}\text { Empeño } \\
\text { Obstáculos } \\
\text { Intimidad } \\
\text { Espíritu de trabajo } \\
\text { Actitud } \\
\text { Acento puesto sobre la producción } \\
\text { Confianza } \\
\text { Consideración }\end{array}$ \\
\hline $\begin{array}{l}\text { Gavin } \\
\text { (1975, pp. 135-139) }\end{array}$ & $\begin{array}{l}\text { Evaluó las relaciones entre } \\
\text { percepciones sobre el ambiente } \\
\text { de trabajo, índices de bienestar } \\
\text { psicológico y de salud. }\end{array}$ & $\begin{array}{l}\text { Estructura organizacional } \\
\text { Obstáculo } \\
\text { Recompensa } \\
\text { Confianza y consideración de parte } \\
\text { de los administrativos } \\
\text { Riesgos y desafios }\end{array}$ \\
\hline $\begin{array}{l}\text { Litwin y Stringer } \\
\text { (1966) }\end{array}$ & $\begin{array}{l}\text { Caracteriza la situación de los } \\
\text { individuos con respecto a sus } \\
\text { motivaciones para el éxito, } \\
\text { las estructuras de poder y } \\
\text { afiliación. }\end{array}$ & $\begin{array}{l}\text { Estructura organizacional } \\
\text { Responsabilidad } \\
\text { Recompensa } \\
\text { Riesgo } \\
\text { Apoyo } \\
\text { Normas } \\
\text { Conflicto }\end{array}$ \\
\hline
\end{tabular}

Pritchard y Karasick (1973, pp. 126-146)

$\operatorname{Moos}(1974)$
La relación del clima organizacional con el resultado de las unidades funcionales y la satisfacción de los individuos en el trabajo.
Autonomía

Conflicto contra la cooperación

Relaciones sociales

Estructura organizacional

Recompensa

Relación entre rendimiento y

remuneración

Niveles de ambición de la empresa

Estatus

Flexibilidad

Centralización

Apoyo

$\begin{array}{ll}\text { Implicación } & \text { Cohesión } \\ \text { Apoyo } & \text { Autonomía } \\ \text { Tarea } & \text { Presión } \\ \text { Claridad } & \text { Control } \\ \text { Innovación } & \text { Confort }\end{array}$


ISSN: 0124-3551 / Año 14, No 21 / julio-diciembre / pp. 247-272

\begin{tabular}{|c|c|c|}
\hline Autor & Propósito & Dimensiones \\
\hline $\begin{array}{l}\text { Schneider y Bartlett } \\
(1968, \text { pp. 323-333) }\end{array}$ & $\begin{array}{l}\text { Diseño de un instrumento } \\
\text { de evaluación de clima } \\
\text { organizacional en puestos } \\
\text { directivos de agencias de } \\
\text { seguros de vida. }\end{array}$ & $\begin{array}{l}\text { Apoyo proveniente de la dirección } \\
\text { Interés por los nuevos empleados } \\
\text { Conflicto } \\
\text { Independencia de los agentes } \\
\text { Satisfacción } \\
\text { Estructura organizacional }\end{array}$ \\
\hline $\begin{array}{l}\text { Downey, Hellriegel, } \\
\text { Phelps y Slocum } \\
\text { (1974, pp. } 233-248)\end{array}$ & $\begin{array}{l}\text { Evaluar la influencia del } \\
\text { clima organizacional en la } \\
\text { satisfacción del individuo y la } \\
\text { ejecución de la tarea. }\end{array}$ & $\begin{array}{l}\text { Toma de decisiones } \\
\text { Calidez } \\
\text { Riesgo } \\
\text { Apertura } \\
\text { Recompensa } \\
\text { Estructura }\end{array}$ \\
\hline $\begin{array}{l}\text { Sims y Lafollette } \\
(1975, \text { pp. } 19-38)\end{array}$ & $\begin{array}{l}\text { Relación entre clima } \\
\text { organizacional y satisfacción. }\end{array}$ & $\begin{array}{l}\text { Presión del trabajo y estándares } \\
\text { Tono general de afecto hacia la } \\
\text { dirección y/o la organización } \\
\text { Riesgo en la toma de decisiones } \\
\text { Política y claridad de promoción } \\
\text { Apertura de la comunicación } \\
\text { ascendente } \\
\text { Tono de afecto hacia otra gente } \\
\text { dentro de la organización }\end{array}$ \\
\hline $\begin{array}{l}\text { Méndez } \\
\text { (2006, pp. 67-72) }\end{array}$ & $\begin{array}{l}\text { Diseño de un instrumento } \\
\text { de clima organizacional para } \\
\text { implementar en universidades } \\
\text { y en el sector productivo } \\
\text { colombiano. }\end{array}$ & $\begin{array}{l}\text { Objetivos } \\
\text { Cooperación } \\
\text { Liderazgo } \\
\text { Toma de decisiones } \\
\text { Relaciones interpersonales } \\
\text { Motivación } \\
\text { Control }\end{array}$ \\
\hline $\begin{array}{l}\text { Toro } \\
(2009, \text { pp. } 45-50)\end{array}$ & $\begin{array}{l}\text { Diseño de un instrumento } \\
\text { de clima organizacional } \\
\text { para ser implementado en } \\
\text { organizaciones de servicios o } \\
\text { del sector productivo a nivel } \\
\text { latinoamericano. }\end{array}$ & $\begin{array}{l}\text { Trato interpersonal } \\
\text { Apoyo del jefe } \\
\text { Sentido de pertenencia } \\
\text { Retribución } \\
\text { Disponibilidad de recursos } \\
\text { Estabilidad } \\
\text { Claridad Organizacional } \\
\text { Coherencia } \\
\text { Valores colectivos } \\
\text { Disposición al esfuerzo }\end{array}$ \\
\hline
\end{tabular}

Fuente: Adaptado de Brunet (1987, pp. 45-52), Silva (1992, pp. 80-127), Santana y Cabrera (2006, pp. 12-18), Méndez (2006, pp. 67-72), Toro (2009, pp. 45-50). 


\section{Caracterización de la universidad objeto de estudio}

La universidad objeto de estudio es de carácter nacional; cuenta con 61 sedes con influencia en todo el territorio colombiano y su rol institucional se orienta al logro de metas y objetivos con un criterio basado en la calidad y la productividad educativa; está perfilada en la consecución de altos niveles de desarrollo de las actividades ejecutadas por los individuos que conforman la organización, acompañada de unos indicadores de calidad, eficiencia, eficacia y efectividad que permitan evaluar los procesos y los resultados de las operaciones; de otro lado, en la entidad se reconoce que un factor primordial para alcanzar sus propósitos es su capital humano, por lo tanto plantea como objetivo institucional el desarrollo de planes de incentivos, actividades de evaluación de desempeño y satisfacción de los empleados, programas para el mejoramiento del clima organizacional, encaminados a motivar a los empleados para el desarrollo de sus actividades con miras a alcanzar los objetivos de la organización. En la estructura formal de la institución se identifican unidades misionales y de gestión, así:

\section{Unidades misionales}

- Las direcciones zonales y de centros de educación.

- Las escuelas académicas.

- Los consejos de escuela.

- Las vicerrectorías de Desarrollo Regional y de Proyección, Académica y de Investigación y de Medios y Mediaciones Pedagógicas.

- Consejo Académico.

- Secretaría General.

\section{Unidades de gestión}

- $\quad$ Sistema Nacional de Talento Humano.

- Las gerencias Administrativa y Financiera, de Relaciones Interinstitucionales, y de Innovación y Desarrollo Tecnológico.

- Consejo Superior Universitario.

- Las oficinas de asesoría jurídica, asesoría de planeación, control interno, control interno disciplinario.

Además, existen dos entes maestros que se encuentran interrelacionados con las unidades misionales y de gestión: la Rectoría y la Oficina de Calidad y Mejoramiento Continúo. 
ISSN: 0124-3551 / Año 14, No 21 / julio-diciembre / pp. 247-272

\section{Desarrollo metodológico}

\subsection{Características del instrumento}

Uno de los propósitos de la universidad objeto de estudio, es el mejoramiento continuo a través de la propuesta de un plan de desarrollo que pretende alcanzar objetivos específicos, determinar una serie de actividades encaminadas en la búsqueda de un mejor ambiente de trabajo y la implementación de una serie de estímulos y recompensas para los individuos que laboran en la institución, por lo anterior es primordial en la investigación la utilización de un instrumento de clima organizacional para evaluar las metas propuestas por la institución, con miras a ofrecer un servicio educativo de calidad y evaluar la percepción de los trabajadores frente a las recompensas y beneficios que ofrece la organización; en este sentido es relevante conocer instrumentos que midan estos aspectos, al respecto Silva (1992, p. 80-127) y Méndez (2006, p. 38), identificaron autores que desarrollaron investigaciones sobre el diseño de instrumentos de clima organizacional orientados hacia la recompensa, en los cuales se abordan diferentes temas, entre otros, los comportamientos en el trabajo, la consecución de logros y metas, la satisfacción del empleado, los beneficios y las recompensas que ofrece la organización, los cuales cumplen con los requerimientos de medición planteados por la organización objeto de estudio. Estos trabajos son punto de referencia para la selección del instrumento a implementar en el estudio. Silva (1992, pp. 80-127) y Méndez (2006, pp. 38), reconocen entre quienes diseñaron instrumentos bajo estos parámetros a Pritchard y Karasick (1973), Moos (1974), Downey, Hellriegel, Phels y Slocum (1974), Sims y Lafollette (1975).

En la tabla 2 se muestran los factores y la conceptualización de las variables incluidas por los autores mencionados.

Tabla 2. Instrumentos de clima orientados a la recompensa

\begin{tabular}{|l|l|l|}
\hline \multicolumn{1}{|c|}{ Autores } & $\begin{array}{c}\text { Variables relacionadas } \\
\text { conceptualmente }\end{array}$ & $\begin{array}{l}\text { Sinopsis de las principales características } \\
\text { que evalúa la variable }\end{array}$ \\
\hline Moss (1974) & Implicación & $\begin{array}{l}\text { Hasta donde los individuos se implican con su } \\
\text { trabajo. }\end{array}$ \\
\hline Moss (1974) & Cohesión & $\begin{array}{l}\text { Relaciones de amistad y apoyo entre los traba- } \\
\text { jadores. }\end{array}$ \\
\hline $\begin{array}{l}\text { Pritchard y Karasick } \\
(1973, \text { pp. 126-146) }\end{array}$ & $\begin{array}{l}\text { Conflicto frente a } \\
\text { cooperación }\end{array}$ & $\begin{array}{l}\text { Cooperación entre los empleados, el desempeño } \\
\text { de sus funciones y el trabajo en equipo. }\end{array}$ \\
\hline & Relaciones sociales & $\begin{array}{l}\text { Compañerismo y calidez entre los miembros de } \\
\text { la organización. }\end{array}$ \\
\hline
\end{tabular}


Metodología de evaluación del clima organizacional a través de un modelo de regresión logística para una universidad en Bogotá, Colombia

Juan Camilo Vega, Edgar Guillermo Rodríguez Díaz, Alexandra Montoya R.

\begin{tabular}{|l|l|l|}
\hline \multicolumn{1}{|c|}{ Autores } & $\begin{array}{c}\text { Variables relacionadas } \\
\text { conceptualmente }\end{array}$ & $\begin{array}{l}\text { Sinopsis de las principales características } \\
\text { que evalúa la variable }\end{array}$ \\
\begin{tabular}{|l|l|} 
Downey, Hellriegel, \\
Phelps y Slocum \\
$(1974$, pp. 233-248)
\end{tabular} & Apertura & $\begin{array}{l}\text { Facilidad o dificultad para establecer } \\
\text { familiaridad y trato con los otros individuos de } \\
\text { la organización. }\end{array}$ \\
\hline $\begin{array}{l}\text { Calidez } \\
(1975, \text { pp. 19-38) }\end{array}$ & $\begin{array}{l}\text { Apertura de la } \\
\text { Comunicación ascendente } \\
\text { nización. }\end{array}$ & $\begin{array}{l}\text { Comunerismo y confianza dentro de la orga- } \\
\text { dirección; tomar las ideas de los subordinados } \\
\text { para actuar. }\end{array}$ \\
\hline & $\begin{array}{l}\text { Tono de afecto hacia } \\
\text { otra gente dentro de la } \\
\text { organización. }\end{array}$ & Percepción, compañeros de trabajo. \\
\hline
\end{tabular}

Moss (1974) Autonomía

Forma en que la organización anima a los trabajadores a ser autónomos y tomar decisiones.

\begin{tabular}{|c|c|c|}
\hline Moss (1974) & Tarea & $\begin{array}{l}\text { Manera en que el clima de trabajo estimula la } \\
\text { planificación y la eficacia en el trabajo de los } \\
\text { individuos dentro de la organización. }\end{array}$ \\
\hline Moss (1974) & Presión & $\begin{array}{l}\text { Es ejercida por la dirección sobre los empleados } \\
\text { para que se lleve a cabo el trabajo. }\end{array}$ \\
\hline Moss (1974) & Claridad & $\begin{array}{l}\text { En los reglamentos y las políticas } \\
\text { organizacionales. }\end{array}$ \\
\hline $\begin{array}{l}\text { Pritchard y Karasick } \\
(1973, \text { pp. 126-146) }\end{array}$ & Estructura organizacional & $\begin{array}{l}\text { La claridad de los métodos, los procesos y las } \\
\text { funciones, así como de las políticas y directrices } \\
\text { de la organización para que los empleados } \\
\text { realicen adecuadamente su trabajo. }\end{array}$ \\
\hline $\begin{array}{l}\text { Sims y Lafollette } \\
(1975, \text { pp. 19-38) }\end{array}$ & $\begin{array}{l}\text { Política y claridad de } \\
\text { promoción. }\end{array}$ & $\begin{array}{l}\text { Percepción de la claridad de las políticas } \\
\text { organizacionales, la estructura organizacional y } \\
\text { la definición del trabajo. }\end{array}$ \\
\hline $\begin{array}{l}\text { Downey, Hellriegel, } \\
\text { Phelps y Slocum } \\
\text { (1974, pp. 233-248) }\end{array}$ & Estructura & $\begin{array}{l}\text { Claridad que perciben los empleados } \\
\text { con respecto a las políticas, la estructura } \\
\text { organizacional física, social y de sus } \\
\text { responsabilidades. }\end{array}$ \\
\hline Moss (1974) & Control & $\begin{array}{l}\text { Se refiere a los reglamentos y a las presiones } \\
\text { que puede utilizar la dirección para controlar a } \\
\text { sus empleados. }\end{array}$ \\
\hline Moss (1974) & Innovación & $\begin{array}{l}\text { Mide la importancia que la dirección puede } \\
\text { dar al cambio y a las nuevas formas de llevar el } \\
\text { trabajo (iniciativa). }\end{array}$ \\
\hline Moss (1974) & Confort & $\begin{array}{l}\text { Mide los esfuerzos que realiza la dirección para } \\
\text { crear un ambiente físico sano y agradable para } \\
\text { los empleados. }\end{array}$ \\
\hline
\end{tabular}




\begin{tabular}{|l|l|}
\multicolumn{1}{|c|}{ Autores } & $\begin{array}{c}\text { Variables relacionadas } \\
\text { conceptualmente }\end{array}$ \\
\hline $\begin{array}{l}\text { Pritchard y Karasick } \\
\text { (1973, pp. 126-146) }\end{array}$ & $\begin{array}{l}\text { Autonomía / Toma de } \\
\text { decisiones (directivos) }\end{array}$ \\
\hline $\begin{array}{l}\text { Pritchard y Karasick } \\
\text { (1973, pp. 126-146) }\end{array}$ & Nivel de recompensas \\
\hline
\end{tabular}

Downey, Hellriegel, Phelps y Slocum (1974, pp. 233-248)

Pritchard y Karasick (1973, pp. 126-146 )

Relación entre rendimiento y remuneración

Pritchard y Karasick (1973, pp. 126-146)

Pritchard y Karasick (1973, pp. 126-146)

Pritchard y Karasick (1973, pp. 126-146)

Pritchard y Karasick (1973, pp. 126-146

Sims y Lafollette (1975, pp. 19-38)

Recompensa

Motivación para el rendimiento

Polarización del estatus

Flexibilidad e innovación

Centralización de decisiones

Apertura de la comunicación ascendente
Riesgo en la toma de decisiones

Downey, Hellriegel, Phelps y Slocum (1974, pp. 233-248)

Sims y Lafollette (1975, pp. 19-38)

Sims y Lafollette (1975, pp. 19-38)

Downey, Hellriegel, Phelps y Slocum (1974, pp. 233-248)

Tono general de afecto hacia la dirección y/o la organización

Presión del trabajo y estándares

Riesgo
Sinopsis de las principales características que evalúa la variable

Grado de autonomía y libertad de los directivos para la toma de decisiones.

Se refiere a la calidad de las recompensas y las retribuciones económicas (salarios), de reconocimiento u otros símbolos de estatus.

Se refiere al sentimiento que los individuos tienen acerca de la importancia del estímulo recibido de la dirección, y del humanitarismo que manifiestan como componentes del sistema de recompensas.

Mide la relación entre el trabajo realizado conforme a las habilidades de cada individuo y la retribución recibida, estableciendo que las recompensas estén basadas en el mérito.

Mide los niveles de ambición de la empresa basada en los aspectos motivacionales que desarrolla la organización en sus empleados.

Se refiere a la importancia que la organización otorga a las diferencias jerárquicas.

Mide el grado de receptividad que tiene la organización a nuevas ideas, métodos y procedimientos.

Mide el grado en que se comparte la autoridad y la toma de decisiones.

Comunicación entre los empleados y la dirección.

Disposición de los directivos a actuar basado en las sugerencias de los subordinados.

Percepciones que los empleados tienen acerca de los procesos de toma de decisiones, la estructura y los estándares que están asociados con estos procesos.

Mide la manera como los miembros de la organización perciben a sus directivos.

Percepción sobre la presión que existe en el trabajo.

Evalúa los estándares implementados en el trabajo.

Sentimiento de reto personal y de toma de riesgo corporativo, asociado con el éxito de la organización.

Fuente: Adaptado de Brunet (1987, pp. 45-52), Silva (1992, pp. 80-127) y Méndez (2006, pp. 67-72). 
Como lo plantean Terrén (2003, pp. 141-144) y Brunet (1987, p. 51), existen diversidad de factores que se miden en los distintos instrumentos diseñados para evaluar clima organizacional, pero se evidencian cuatro elementos básicos de evaluación comunes dentro de los distintos cuestionarios: la autonomía individual, el grado de estructura organizacional, los tipos de recompensa y la consideración; cada investigador basado en estos elementos y los objetivos del estudio, diseña y define las variables a incluir dentro del cuestionario para medir el clima organizacional (Terrén, 2003, pp. 141-144). Para la selección del instrumento se tomó un primer parámetro que incluyera los cuatro factores básicos para evaluar clima y que la construcción de las variables se orientará a evaluar la recompensa o la motivación con el fin de alcanzar los objetivos de la organización.

Con base en los factores expuestos se seleccionó un instrumento orientado a evaluar clima organizacional ${ }^{1}$, este cuestionario se adaptó a la estructura organizacional de la universidad así como al lenguaje institucional, validándolo posteriormente con el personal docente. En él también se solicitó información relacionada con formación, género, edad, cargo, tiempo de servicio, tipo de contrato, ubicación funcional y de sede. El instrumento tiene una confiabilidad del $90 \%$ y consta de 58 ítems enunciados positiva y negativamente con respuesta alternativa de verdadera o falsa y evaluando siete dimensiones, cada una contiene un par de preguntas de control. En la tabla 3 se presenta la conceptualización de las dimensiones.

\section{Metodología}

El estudio se realizó con el personal administrativo y académico que labora en las diferentes unidades misionales y unidades de gestión en las dos sedes (Central y Sede), ubicadas en la ciudad de Bogotá, Colombia, vinculado durante el primer periodo de 2010 a la universidad, bajo cualquiera de las modalidades que maneja la institución (planta, resolución y orden de prestación de servicios).

La aplicación del cuestionario se realizó de forma física y directa al personal en las dos sedes de la institución entre quienes, de forma voluntaria, accedieron a diligenciarlo; el instrumento de evaluación de clima organizacional fue entregado de forma individual a cada uno de los participantes en su lugar de trabajo, e inmediatamente después de su devolución se comprobó la veracidad de datos con respecto a ubicación funcional y sede, siendo estos los aspectos a comparar en el estudio.

1 Se analizaron dos posibilidades de instrumentos para evaluar el clima organizacional: los trabajos elaborados para optar por el título de psicología de la Universidad Nacional de Colombia. Al hacer la revisión se identificó que una de las tesis aborda un enfoque orientado a medir la influencia del liderazgo en el clima organizacional, y la otra busca la relación frente a la motivación y la recompensa fundamentando su diseño en los cuatro elementos básicos para evaluar clima organizacional; con estos antecedentes se seleccionó la investigación denominada Diagnóstico de clima organizacional mediante el diseño y análisis de una encuesta para medir ambiente laboral (Otálora, 1993). 
ISSN: 0124-3551 / Año 14, No 21 / julio-diciembre / pp. 247-272

Tabla 3. Variables del instrumento

\section{Denominación}

Calidad

Distinción

Responsabilidad

Salario

Orden organizacional

Apoyo laboral

Seguridad

\section{Definición de variables}

Indica el grado en que los objetivos y funciones que fija la empresa constituyen un reto para el trabajador y le permiten progresar a través del logro de resultados y del mejoramiento de sus destrezas, conocimientos y actitudes. Se relaciona con el "grado de estructura organizacional".

Indica si el trabajo de los miembros de la organización es elevado o no, y en consecuencia estos son admirados y recompensados cuando la calidad del trabajo es buena, o sancionados y criticados cuando no lo es (existencia de equidad), o en el peor de los casos, son ignorados sin importar los resultados de su trabajo. Se relaciona con "consideración agradecimiento- apoyo".

Mide el grado en que la organización asigna deberes a sus miembros y cómo responden ante los mismos con presencia de iniciativa y capacidad laboral. Se relaciona con "autonomía individual".

Indica el aspecto remunerativo que ofrece la organización, tiene en cuenta la calidad de los sueldos, la capacidad adquisitiva de los mismos y se mide en comparación con otras organizaciones. Se relaciona con "tipo de recompensa".

Señala la forma en que la organización planifica sus estructuras física (recurso técnico) y social (recurso humano). Esta dimensión es importante para aplicar programas de acción. Se relaciona con "grado de estructura organizacional".

Muestra el grado de posibilidades reales que la organización da al trabajador para intervenir en la solución de problemas laborales, establecimiento de políticas etc., y a su vez mide el nivel de autonomía que la institución ofrece al individuo para decidir cómo realizar su trabajo. Se relaciona con "agradecimiento - consideración".

Mide la escala de comodidad y aceptación que el trabajador posee de la organización y a su vez, define las reales características y los elementos que esta ofrece a cada individuo. Se relaciona con los cuatro elementos básicos para la elaboración de instrumento de clima organizacional.

Fuente: Adaptado de Otálora (1993).

Se entregaron 289 cuestionarios para ser diligenciados y fueron resueltos 169. Se realizó un primer filtro de las encuestas eliminando las que tenían cuatro ítems o más sin diligenciar de esta manera fueron eliminados 13, luego los cuestionarios que tenían inconsistencias de respuesta iguales o superiores a cinco (más de cinco pares de respuestas de control contradictorias), excluyendo 10 encuestas, por esta razón se contó con una base de estudio de 146 cuestionarios. 
Metodología de evaluación del clima organizacional a través de un modelo de regresión logística para una universidad en Bogotá, Colombia

Juan Camilo Vega, Edgar Guillermo Rodríguez Díaz, Alexandra Montoya R.

La primera evaluación del clima organizacional se perfiló en la medición de clima por variable, a través de un análisis de frecuencia de calificación. Se filtraron los ítems correspondientes a cada una de las dimensiones, y se tomaron como referencia para evaluar cada variable solo los cuestionarios en los que se diligenciaron la totalidad de los ítems por dimensión, arrojando la siguiente base de cuestionarios por aspecto a evaluar, calidad, 132; distinción, 124: responsabilidad, 131; salario, 115; orden organizacional, 126; apoyo laboral, 131; seguridad, 119.

Luego se consolidaron las calificaciones de cada una de las dimensiones en estudio; para efectos prácticos del análisis de puntajes obtenidos fue necesario elaborar una escala de evaluación que permitió ponderar las calificaciones y eliminar los efectos de calificaciones atípicas, se diseñó una escala de puntuación basada en la escala Stanine (estándar nine), la cual asume una distribución de los datos de forma normal (Z) y divide dicha distribución en nueve intervalos con una media $=5$ y desviación estándar $=$ 2, (Monroy y Pedraza, 1990). Los resultados de Stanine de 1, 2, ó 3 indican un funcionamiento por debajo del promedio. Los "stanines" de 4, 5 y 6 se consideran promedio, y los "stanines" de 7, 8 ó 9, arriba del promedio (Wayne, 2004). La distribución de la escala de calificación que se aplica al estudio² es la siguiente:

Tabla 4. Escala de evaluación de resultados

\begin{tabular}{|c|c|c|}
\hline \multicolumn{1}{|c|}{ Puntaje Z } & Puntación escala Stanine & Percepción \\
\hline+1.76 o más & 9 & Excelente \\
\hline$+1.26 \mathrm{a}+1.75$ & 8 & Muy buena \\
\hline$+0.76 \mathrm{a}+1.25$ & 7 & Muy buena \\
\hline$+0.26 \mathrm{a}+0.75$ & 6 & Buena \\
\hline$-.0 .25 \mathrm{a}+0.25$ & 5 & Buena \\
\hline$-0.75 \mathrm{a}-0.26$ & 4 & Buena \\
\hline-1.25 a -0.76 & 3 & Baja \\
\hline$-1.75 \mathrm{a}-1.26$ & 2 & Baja \\
\hline-1.76 o menos & 1 & Muy baja \\
\hline
\end{tabular}

Fuente: Adaptado de Wayne (2004).

2 En la escala de evaluación de clima se decidió adicionar dos rangos a los establecidos por Wayne (2004), excelente y muy baja, en la medida en que para el estudio es importante separar los rangos de calificación extremos. 


\section{ĊIF́E 21}

ISSN: 0124-3551 / Año 14, No 21 / julio-diciembre / pp. 247-272

Finalmente, las frecuencias obtenidas se estandarizan a los valores de la distribución de probabilidades normal estándar $(\mathbf{Z})^{3}$, mediante:

$$
\mathrm{Z}=\frac{\mathrm{X}-u}{\delta}
$$

La siguiente evaluación del clima organizacional en la universidad se orientó en dos cohortes: la primera enfocada en la comparación de las dimensiones de clima organizacional de acuerdo con la sede donde labora el funcionario; y la segunda, cotejando las dimensiones de clima organizacional según la unidad funcional a la cual pertenece el empleado. Para los dos escenarios en cuestión se procedió a eliminar de la totalidad del consolidado de cuestionarios aquellos en los cuales se dejó de diligenciar por lo menos un ítem, o no se estableció la ubicación organizacional para la primera cohorte, o no se identifica la unidad funcional para el caso de la segunda cohorte. Obteniendo la siguiente base de cuestionarios, cohorte ubicación organizacional, 90; cohorte unidad funcional; 87.

La evaluación de las dos cohortes busca determinar la asociación de cada una de las dimensiones de clima con respecto a ubicación organizacional y unidad funcional y a partir de allí inferir diferencias y particularidades que permitan contrastar los escenarios, para lograrlo se implementó un modelo de regresión logística que permitiera la obtención de los parámetros de interés de la investigación.

La regresión logística es un modelo estadístico que permite estudiar si una variable binomial depende, o no, de una u otras variables no necesariamente binomiales, (Hosmer y Lemeshow 1989), se dice que una variable es binomial cuando tiene dos posibles resultados: éxito o fracaso. Los modelos de regresión logística cuentan con contrastes estadísticos directos, capacidad para incorporar efectos no lineales y permitir una amplia variedad de diagnósticos. Los propósitos de este modelamiento estadístico son de tipo estimativo y predictivo. El modelo asume que la variable dependiente $(\mathrm{Y})$ es binaria, para lo cual tomará valores de: $\mathrm{Y}=1$ a la "presencia" del evento y $\mathrm{Y}=0$ a la "ausencia" del evento, utiliza la siguiente función logística:

$$
\begin{gathered}
h(z)=\frac{1}{1+e^{-z}} \text { Donde } \\
z=\beta_{0}+\beta_{1} X_{1}+\beta_{2} X_{2}+\ldots+\beta_{k} X_{k}
\end{gathered}
$$

Donde $X_{1}, X_{2}, \ldots, X_{k}$ son covariables y la probabilidad de pertenecer a una población se calcula utilizando la siguiente función:

3 Donde $\mathrm{X}=$ Observación tomada de la población o muestra, $\sigma=$ Desviación estándar, y $\mu=$ Media de la población. 
Como

$$
p_{i}=\frac{1}{1+e^{-z}}
$$

$$
1-p_{i}=\frac{1}{1+e^{z}}
$$

Para los estudios que tienen como fin identificar asociación entre variables, siendo el objetivo del estudio planteado; determinar la incidencia de ciertas variables sobre un hecho específico o para aquellos estudios de tipo descriptivo, utilizar el modelamiento estadístico a través de la regresión logística resulta ser muy útil ya que permite el análisis de las variables así estas no sean de tipo discreto o continuo, permitiendo el análisis de covariables de tipo cualitativo ya sean nominales u ordinales; debido a ello el interés del estudio se centra más en la calidad de la asociación de las variables (siete dimensiones que evalúa el instrumento de clima) sobre las cuales se modifica el comportamiento de la variable en estudio (clima organizacional) por lo que es relevante explicar los parámetros del modelo.

\subsection{Parámetros del modelo}

Observando la estructura del modelo de regresión logística

Donde

$$
h(z)=\frac{1}{1+e^{-z}}
$$

$$
z=\beta_{0}+\beta_{1} X_{1}+\beta_{2} X_{2}+\ldots+\beta_{k} X_{k}
$$

Los coeficientes de cada una de las variables $\beta_{i}$ conocidos con el término odds y que se definen como la razón entre la probabilidad de que un determinado suceso ocurra y la probabilidad de que no. Es decir, un número que representan cuánto es más probable que el hecho ocurra frente a que no se produzca. De otro lado, $\beta_{n}$ es el logaritmo de los odds cuando la variable independiente es cero:

$$
\boldsymbol{\beta}_{0}=\ln (p / q \mid X=0)
$$

Mientras que para el valor $\mathrm{X}=1$ :

$$
\ln (p / q \mid X=1)=\boldsymbol{\beta}_{0}+\boldsymbol{\beta}_{1}=\ln (p / q \mid X=0)+\boldsymbol{\beta}_{1}
$$

Por lo tanto, 
ISSN: 0124-3551 / Año 14, No 21 / julio-diciembre / pp. 247-272

$$
\boldsymbol{\beta}_{1}=\ln \frac{\ln (p / q \mid x=1)}{n(p / q \mid x=0)}
$$

Es decir $\beta_{i}$ es el logaritmo del cociente de los odds para los dos valores de la variable $\mathrm{X}$. Si la variable binomial $(\mathrm{Y})$ es independiente de la variable $\mathrm{X}$, ambos odds son iguales, por lo tanto el odds ratio es 1 y su logaritmo será cero. De esta manera, para estudiar con un modelo logístico la independencia de las variables, basta con estudiar si el coeficiente $\beta_{i}$ es cero. Para el caso particular, por ser un estudio con variables determinadas y de carácter asociativo, el foco es analizar los odds ratio $(\mathrm{OR})$ que se obtengan de cada dimensión para cada cohorte e inferir diferencias por sedes para el primer caso y comparaciones por unidad funcional para el segundo, los OR representan cómo aumenta la probabilidad de que suceda el hecho bajo estudio si se presenta un cambio de d unidades en la variable predictora; el OR se obtiene de la siguiente forma:

$$
\begin{gathered}
\eta\left(X_{i}\right)=\beta_{0}+\beta_{1} \chi_{i} \\
\eta\left(X_{i}+1\right)=\beta_{0}+\beta_{1}\left(X_{i}+1\right)
\end{gathered}
$$

Donde:

$\eta\left(X_{i}\right)=$ Es el logaritmo de odds cuando la variable represora es igual a $\left(X_{i}\right)$,

$\eta\left(X_{i}+1\right)=$ Es el logaritmo de odds cuando la variable regresora es igual a $\left(X_{i}+1\right)$.

La diferencia entre los valores predichos será:

$$
\begin{gathered}
\eta\left(X_{i}\right)-n\left(X_{i}+1\right)=\beta_{1} \\
\eta\left(X_{i}\right)-n\left(X_{i}+1\right)=\ln \left(\text { odds } X_{i}+1\right)-\ln \left(\text { odds } X_{i}\right) \\
\text { OR }=\left\langle\frac{\operatorname{odds} X_{i}^{+1}}{\operatorname{odds} X_{i}}\right\rangle=e^{\beta_{1}}
\end{gathered}
$$

Con lo cual se puede concluir que la cantidad exponente $\beta_{j}$ es el cociente de odds del regresor $X_{j}$, suponiendo que las demás variables predictoras permanecen constantes. Los $B$ son los coeficientes de cada una de las variables independientes (Hosmer y Lemeshow 1989). 


\subsection{Modelo teórico para la cohorte ubicación organizacional}

$$
\ln \left(\frac{\rho_{i}}{1-\rho_{i}}\right)=\beta_{1} X_{1}+\beta_{2} X_{2}+\beta_{3} X_{3}+\beta_{4} X_{4}+\beta_{5} X_{5}+\beta_{6} X_{6}+\beta_{7} X_{7}
$$

Donde: Variable dependiente $(\mathrm{Y})=$ Ubicación organizacional (Administrativa $=1$, Carrera $30=0)^{4}$ y las variables independientes son:

$$
\begin{aligned}
& X_{1}=\text { Apoyo Laboral } \\
& X_{2}=\text { Salario } \\
& X_{3}=\text { Calidad } \\
& X_{4}=\text { Seguridad } \\
& X_{5}=\text { Distinción } \\
& X_{6}=\text { Orden organizacional } \\
& X_{7}=\text { Responsabilidad }
\end{aligned}
$$

\subsection{Modelo teórico para la cohorte unidad funcional:}

$$
\ln \left(\frac{\rho_{i}}{1-\rho_{i}}\right)=\beta_{1} X_{1}+\beta_{2} X_{2}+\beta_{3} X_{3}+\beta_{4} X_{4}+\beta_{5} X_{5}+\beta_{6} X_{6}+\beta_{7} X_{7}
$$

Donde: Variable dependiente $(\mathrm{Y})=$ Unidad funcional $(\text { Misional }=1 \text {, Gestión }=0)^{5}$, y las variables independientes son:

$$
\begin{aligned}
& X_{1}=\text { Apoyo Laboral } \\
& X_{2}=\text { Salario } \\
& X_{3}=\text { Calidad } \\
& X_{4}=\text { Seguridad } \\
& X_{5}=\text { Distinción } \\
& X_{6}=\text { Orden organizacional } \\
& X_{7}=\text { Responsabilidad }
\end{aligned}
$$

4 En este punto es de anotar que para el análisis de los parámetros obtenidos por variable ( $\beta$ ), están soportados frente a la presencia del evento de pertenecer a la sede Administrativa.

5 La plataforma de análisis de los parámetros obtenidos por variable $(\boldsymbol{\beta})$, se fundamentan frente a la presencia del evento de pertenecer a unidades misionales. 


\section{Análisis de resultados}

En el análisis de frecuencias acumuladas por variable ( tabla 5) se aprecia como perfil de interés que la frecuencia acumulada de calificación media Stanine en todas las variables está por encima del $65 \%$, llamando la atención que la dimensión con menor porcentaje de frecuencias superior a la media es la de salario (66\%), lo cual permite inferir que a nivel general, dentro de la organización, existe un buen clima organizacional, de otro lado es de recalcar que los mayores porcentajes de frecuencia acumulada inferiores a la media de calificación se ubican en las variables: salario (34\%), distinción (29 \%) y orden organizacional (24\%); un primer indicador sobre las dimensiones que serian susceptibles a ejercer acciones de mejoramiento en la institución.

Tabla 5. Resultados escala Stanine

\begin{tabular}{|c|c|c|c|c|c|c|c|c|}
\hline Variable & $\mathbf{N}$ & 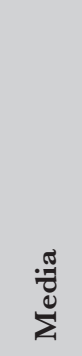 & 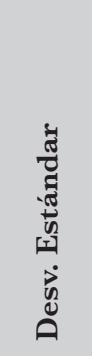 &  & 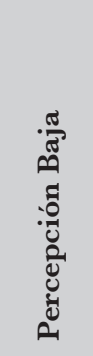 & 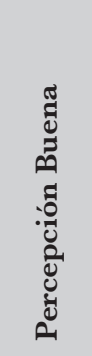 & 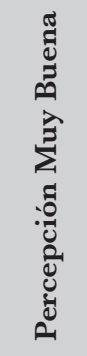 &  \\
\hline Apoyo laboral & 131 & 6,85 & 1.555 & $9 \%$ & $7 \%$ & $76 \%$ & $8 \%$ & \\
\hline Salario & 115 & 3,91 & 2.261 & $2 \%$ & $32 \%$ & $33 \%$ & $32 \%$ & $1 \%$ \\
\hline Calidad & 132 & 6,35 & 1.807 & $9 \%$ & $7 \%$ & $50 \%$ & $34 \%$ & \\
\hline Seguridad & 119 & 4,92 & 1.619 & $7 \%$ & $13 \%$ & $62 \%$ & $17 \%$ & $2 \%$ \\
\hline Distinción & 124 & 3,81 & 1.886 & $3 \%$ & $26 \%$ & $44 \%$ & $27 \%$ & \\
\hline Orden Organizacional & 126 & 5,4 & 2.235 & $8 \%$ & $16 \%$ & $56 \%$ & $21 \%$ & \\
\hline Responsabilidad & 131 & 8,35 & 1.779 & $8 \%$ & $10 \%$ & $48 \%$ & $34 \%$ & \\
\hline
\end{tabular}

Los resultados de la aplicación del modelo de regresión logística por unidad funcional (tabla 6) determinan líneas de acción más específicas sobre los aspectos identificados en el análisis de frecuencias, que permitirán surtir más efecto y un menor desgaste en la 
Metodología de evaluación del clima organizacional a través de un modelo de regresión logística para una universidad en Bogotá, Colombia

Juan Camilo Vega, Edgar Guillermo Rodríguez Díaz, Alexandra Montoya R.

organización con miras a mejorar el ambiente laboral, al respecto es necesario recalcar que los trabajadores misionales califican mucho mejor sus percepción frente al salario (calificaciones 1,2 veces más altas que las del personal de gestión), lo cual indica que se deben focalizar las acciones de remuneración sobre el personal de gestión; en la dimensión de distinción se aprecia que los empleados que desarrollan labores de gestión perciben que su trabajo es más reconocido frente a los misionales, siendo interesante este punto pues los misionales a pesar de percibir que son bien remunerados denotan que falta un mayor reconocimiento de su labor dentro de la organización; caso contrario sucede con el personal de gestión.

En lo concerniente al orden organizacional, los trabajadores misionales perciben menor planificación con respecto a la disposición de recursos físicos y humanos por parte de la organización, hecho para resaltar pues son estos quienes ejecutan el objetivo primordial de la institución.

Tabla 6. Evaluación de clima organizacional por unidad funcional

\begin{tabular}{|c|c|c|}
\hline UNIDAD & N & \\
\hline GESTIÓN & $\mathbf{2 8}$ & \\
\hline MISIONAL & $\mathbf{5 9}$ & \\
\hline Total & $\mathbf{8 7}$ & $\mathbf{O R}$ \\
\hline Dimensión & $\mathbf{B}$ & 1.742 \\
\hline Apoyo laboral & .555 & 1.285 \\
\hline Salario & .250 & 1.451 \\
\hline Calidad & .372 & .840 \\
\hline Seguridad & -.174 & .587 \\
\hline Distinción & -.532 & .856 \\
\hline Orden Organizacional & & \\
\hline Responsabilidad & & \\
\hline
\end{tabular}

Fuente: Elaboración propia.

Los resultados de comparación de clima organizacional por sedes (tabla 7), indican que los trabajadores que desarrollan sus funciones en la unidad física de la sede 


\section{CEIFF'E 21}

ISSN: 0124-3551 / Año 14, No 21 / julio-diciembre / pp. 247-272

administrativa perciben que su trabajo es más reconocido (distinción, calificaciones 1,2 veces más altas que las del personal de la sede carrera 30 ), se observa una tendencia en este grupo de funcionarios a percibir que la labor que desarrollan mejora sus habilidades y destrezas (calidad, calificaciones 1,1 veces más altas que las del personal de la sede carrera 30), sin embargo los trabajadores de la primera, perciben una menor autonomía y remuneración frente a los de la carrera 30 (apoyo laboral y salario con OR por debajo de 1).

Tabla 7. Evaluación de clima organizacional por sede

\begin{tabular}{|l|c|c|}
\hline \multicolumn{1}{|c|}{ Sede } & N & \\
\hline \multicolumn{1}{|c|}{ JAG } & 37 & \\
\hline \multicolumn{1}{|c|}{ JCM } & 53 & \\
\hline \multicolumn{1}{|c|}{ Total } & $\mathbf{9 0}$ & \\
\hline \multicolumn{1}{|c|}{ Dimensión } & $\mathbf{B}$ & OR \\
\hline Apoyo laboral & -.423 & .655 \\
\hline Salario & -.633 & .531 \\
\hline Calidad & .125 & 1.134 \\
\hline Seguridad & .456 & 1.578 \\
\hline Distinción & .473 & 1.605 \\
\hline O. organizacional & .179 & 1.196 \\
\hline Responsabilidad & -.004 & .996 \\
\hline
\end{tabular}

Fuente: Elaboración propia

\section{Conclusiones}

A nivel general un análisis de frecuencias permite calificar el ambiente de trabajo global y de cada uno de los factores evaluados en el instrumento diseñado para tal fin; al utilizar el modelo de regresión logística se complementa la caracterización del clima organizacional y permite evidenciar diferencias significativas con respecto al ambiente laboral en los distintos grupos de interés dentro de la organización, lo cual facilita la toma de decisiones eficientes. 
El estudio de caso en una universidad pública permite validar que existen bajas calificaciones de percepción, mediante el análisis de frecuencia, en tres de las variables de evaluación: salario, distinción y orden organizacional; al utilizar el modelo de regresión logística se aprecian contrastes de percepción entre miembros de distintas unidades funcionales dentro de la organización con respecto a las variables mencionadas, específicamente en lo referente a salarios y reconocimiento, donde el personal docente se percibe bien remunerado pero con una bajo reconocimiento dentro de la organización frente al personal que labora en actividades administrativas. De otro lado, como lo manifiestan diversos estudios de clima organizacional, en este trabajo se evidencian diferencias significativas de percepción entre los individuos por su nivel de estudios, otro perfil que sobresale de los resultados, es la mejor apreciación de clima de los miembros que laboran en la sede donde residen los organismo de poder o de orden estratégico resaltándose sus mejores calificaciones en las dimensiones de pertenencia y reconocimiento.

Finalmente, se puede inferir que en la institución existen diferentes estilos de liderazgo, soportado en las diferencias significativas concernientes al apoyo laboral y la distinción entre sedes y unidades funcionales.

En síntesis, la aplicación de un modelo de regresión logística, demuestra que esta herramienta complementa y enriquece el análisis de resultados con la ventaja de permitir comparaciones de carácter dicotómico e identificar la intensidad de la relación entre los factores evaluados dentro de los distintos grupos de interés establecidos por el investigador.

\section{Referencias}

Alcántar E., Maldonado-Radillo, S., Arcos, J. (2012). Workplace Environment Measurement Essential to Ensure the Effectiveness of Management Quality System. Revista International Administración y Finanzas, 5(3) pp. 55-68. Available at SSRN: http:/ / ssrn.com/abstract $=1954030$

Ashkanasy, N. (2008). Organizational climate. In Stewart R. Glegg and James R. Bailey (Ed.), International encyclopedia of organization studies pp. 1028-1030. Thousand Oaks, California: Sage Publications.

Brunet, L. (1987). El clima de trabajo en las organizaciones. México: Editorial Trillas.

Calderón, G., Álvarez, C. M., Naranjo, J. C. (2006). Gestión Humana en las Organizaciones un Fenómeno Complejo: Evolución, Retos, Tendencias y Perspectivas de Investigación. Cuadernos de Administración, 19(32) pp. 225-254. 


\section{CIFFE 2I}

ISSN: 0124-3551 / Año 14, No 21 / julio-diciembre / pp. 247-272

Caligiori, I., Díaz, J. (2003). Clima Organizacional y Desempeño de los Docentes en la ULA: Estudios de caso. Revista Venezolana de Gerencia. Octubre-Diciembre. 8(24) pp. 644-658. Maracaibo, Venezuela.

Downey, H. K., Hellriegel, D., Phels, M., Slocum, J. W. (1974). Organizational climate and job satisfaction: A comparative analysis, $\mathrm{j}$. of Business Research, 2. pp. 233-248

Forehand, G., Gilmer, B. (1964). Environmental variation in studies of organizational behavior: Psychological Bulletin, 62 pp. 361-382.

Friedlander, F., Margulies, N. (1969). Multiple impacts of organizational climate and individual value systems upon job satisfaction, Personnel Psychology, 22, pp. 171-183.

García, G. (2007). Clima organizacional hacia un nuevo modelo. Revista PORIK $A \mathrm{~N}$, pp. 151-177. Recuperado en: http://www.unicauca.edu.co/porik_an/ imagenes_3noanteriores/No.12porikan/articulo6.pdf

Gavin, J. P. (1975). Organizational climate as a function of personal and organizational variables, j. of Applied psychology, 60, pp. 135-139.

Goncalves, A. (2010). Dimensiones del clima Organizacional. http://www.educadormarista. $\mathrm{com} /$ proyectoaprender/clima-organizacional.htm

Hosmer D., Lemeshow S. (1989). Regresión Logística Aplicada. Nueva York: Editorial Wiley.

Hutt, G., Marmiroli, B. (2007). Estructura Organizacional. Universidad Pedagógica de Durango, México.

Litwin, G. H., Stringer, R. A. (1966). The influence of organizational climate. Boston: Harvard University Press.

Marín, M. (2003). Relación entre el clima y el compromiso organizacional en una empresa del sector petroquímico. (Tesis de pregrado) Escuela de Ciencias Sociales. Universidad Católica Andrés Bello. Caracas, Venezuela.

McMurray, A., Scott, D. (2003). The relationship between organizational climate and organizational culture. Fournal of American Academy of Business, Cambridge, 3(1) pp. 1-8.

Méndez, G. (2006). Clima organizacional en Colombia. Bogotá: Universidad del Rosario.

Monroy, M., Pedraza, F. (1990). Diseño y aplicación de un instrumento para medir clima organizacional. (Tesis) Facultad de Ciencias Humanas. Universidad Nacional de Colombia, Bogotá. 
Metodología de evaluación del clima organizacional a través de un modelo de regresión logística para una universidad en Bogotá, Colombia

Juan Camilo Vega, Edgar Guillermo Rodríguez Díaz, Alexandra Montoya R.

Moos, R. H. (1974). Systems for the assessment and classification of human environments: An overview, R. H. Moos y P.M. Insel (eds.), Issues in social ecology, National Press Books. Palo Alto.

Otalora, D. (1993). Diagnostico de Clima Organizacional Mediante el Diseño y Análisis de una Encuesta para Medir Ambiente Laboral. (Tesis) Facultad de Ciencias Humanas. Universidad Nacional de Colombia, Bogotá.

Pérez, F., Tejada, E. Cuquet, P., Contreras D. (2007). Satisfacción laboral y clima organizacional. Recuperado en: http://www.ucm.es/info/teamwork/abarrasa/ tea/200607_25966/TPGA_G14_Clima.pdf

Pérez, I., Maldonado, M. y Bustamante, S. (2006). Clima organizacional y gerencia: Inductores del cambio organizacional. Investigación y Postgrado, 21(002) pp. 231-248.

Pritchard, R. P., Karasick, B. W. (1973). The effects of organizational climate on managerial job performance and job satisfaction. Organizational behavior Human Performance, 9 pp. $126-146$.

Salinas, C., Laguna, J., Mendoza, M. (1994). La satisfacción laboral y su papel en la evaluación de la calidad de la atención médica. Salud Pública, 36(1) pp. 22-29.

Santana, P. y Cabrera Y. (2006). ¿Dos constructos para explicar un mismo fenómeno? Recuperado en: http://dialnet.unirioja.es/servlet/fichero_articulo? codigo=2486886

Schneider, B., Bartlett, C. J. (1968). Individual differences and organizational climate I: The research plan and questionnaire development. Personnel psychology, 21 pp. 323-333.

Schneider, B., Reichers, A. E. (1983). On the etiology of climates. Personnel psychology, 36 pp. 19-39.

Segredo, A., Pérez L. (2007). El Clima organizacional en el desarrollo de los Sistemas Organizativos. Revista INFODIR. 2007 (4). Recuperado en: http://www.sld.cu/galerias/doc/sitios/infodir/clima_organizacional_en_el_desarrollo_de_sistemas_organizativos.doc

Silva, M. (1992). El clima en las organizaciones. Teoría, método e intervención. Barcelona: Editorial Promociones y Publicaciones Universitarias S.A.

Sims, H. P. Jr., Lafollette, W. (1975). An assessment of the Litwin and stringer Organizational Climate questionnaire. Personnel Psychology, 28 pp. 19-38. 


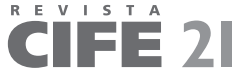

ISSN: 0124-3551 / Año 14, No 21 / julio-diciembre / pp. 247-272

Terrén, E. (2003). Rutina, diversidad e incertidumbre: la organización educativa ante entornos multiculturales. Río de Janeiro: DP \& A.

Toro, A. G. (2009). El clima organizacional. Medellín: Cincel Ltda.

Ucros, M. (2011) Factores del clima organizacional en las universidades de la Costa Caribe Colombiana. Revista Omnia, Año 17 (2) pp. 91 - 102. Universidad del Zulia. ISSN: 1315-8856.

Wayne, M.D. (2004). Bioestadística: Bases para el análisis de las ciencias de la salud. México: Editorial Limusa. 